\title{
Small Molecules, Big Promises: Improvement of Psoriasis Severity and Glucidic Markers with Apremilast: A Case Report
}

This article was published in the following Dove Press journal: Diabetes, Metabolic Syndrome and Obesity: Targets and Therapy

\author{
Caterina Lanna $\mathbb{D}^{*}$ \\ Gaia Maria Cesaroni $\mathbb{D}^{*}$ \\ Sara Mazzilli \\ Luca Bianchi \\ Elena Campione (iD \\ Dermatologic Unit, University of Rome \\ Tor Vergata, Rome, Italy \\ *These authors contributed equally to \\ this work
}

\begin{abstract}
Psoriasis is a common inflammatory skin condition frequently associated with cardiometabolic diseases such as diabetes. Indeed, the state of systemic inflammation typical of psoriasis leads to an increase in the level of IL-1, IL-6 and TNF-alpha which may cause a reduced sensitivity to insulin and, ultimately, can lead to type 2 diabetes mellitus. Particularly, the derangement of PDE4-cAMP signaling has a critical role in disordered glucose and lipid metabolism. Apremilast, as a selective inhibitor of PDE4-cAMP signalling, represents an innovative therapeutic strategy for psoriasis. Here, we report a case of a patient affected by psoriasis and diabetes, who - after using Apremilast - improved his glucose metabolism as well as his need of anti-diabetic drugs and his psoriasis. This suggests that, in addition to its role against psoriasis, Apremilast may even act as a metabolic modulator.

Keywords: psoriasis, diabetes, PDE4-inhibitor
\end{abstract}

\section{Introduction}

Psoriasis is a common inflammatory skin condition, involving $2-4 \%$ of the global population. ${ }^{1}$ It is clinically characterized by erythematous and scaly plaques of variable severity, which have a strong impact on the quality of life of the affected individuals. ${ }^{2}$ The onset of this pathology is due to the interaction between genetic, environmental and immunological factors; thus, it is classified as an immunomediated inflammatory disease (IMID), chronic disease deriving from immunological de-regulation. Recent studies show that psoriasis can be associated with comorbidities, which have systemic inflammation as their predisposing factor, especially in patients with moderate-severe psoriasis. $^{3}$

Indeed, numerous metabolic pathologies correlate with a state of abnormal and chronic inflammatory response and are characterized by excessive production of proinflammatory cytokines and acute phase proteins. ${ }^{4}$ Inflammatory mediators are produced by different types of cells including macrophages and adipocytes. ${ }^{5}$ The state of systemic inflammation associated with psoriatic pathology leads to an increase in the level of IL-1, IL-6 and TNF-alpha, which may cause a reduced sensitivity to insulin and, ultimately, can lead to type 2 diabetes mellitus (DM2).

Particularly, the derangement of the phosphodiesterase 4 (PDE4) and cyclic monophosphate adenosine (cAMP) signaling, highly expressed by cells involved in the regulation of the immune response, has a critical role in disordered glucose and lipid metabolism as well as in hepatic steatosis, abnormal lipolysis, suppressed thermogenic function and deranged neuroendocrine functions. ${ }^{6}$
Correspondence: Caterina Lanna Dermatologic Unit, Department of Systems Medicine, University of Rome Tor Vergata, Viale Oxford 8I, Rome 00I33, Italy

Email caterinalanna.cl@gmail.com 
Apremilast, as a selective inhibitor of PDE4-cAMP signalling, represents an innovative therapeutic strategy for psoriasis, in case of failure, contraindication or intolerance to other systemic treatments such as DMARDs or biologic therapies. ${ }^{7}$

Here we report a case of a patient affected by psoriasis and diabetes, who - after using Apremilast - improved his glucose metabolism as well as his need of anti-diabetic drugs and his psoriasis. This suggests that, in addition to its role against psoriasis, Apremilast may even act as a metabolic modulator.

\section{Case Report}

A 55-year-old man with a history of blood hypertension, diabetes and dyslipidaemia referred to our department.

He had a 20-year history of plaque psoriasis, previously treated with methotrexate, interrupted due to secondary ineffectiveness and Adalimumab, interrupted for infectious complications (Hepatitis E infection). Physical examination showed the presence of diffuse pink-red scaly plaques on the trunk, buttocks, periumbilical areas, dorsal hands and upper and lower extremities involving 30\% body surface area, with a Psoriasis Area Severity Index score of 18 (Figure 1). The patient did not report any family history of psoriasis and said he had no pains in his joints. DNA analysis showed the following haplotype: HLA-A*02, A*30-B*13, $\mathrm{B}^{*} 41-\mathrm{C}^{*} 06, \mathrm{C}^{*} 17$; $\mathrm{C}^{*} 12, \mathrm{C}^{*} 17$. The patient's clinical condition negatively affected his quality of life, with a DLQI

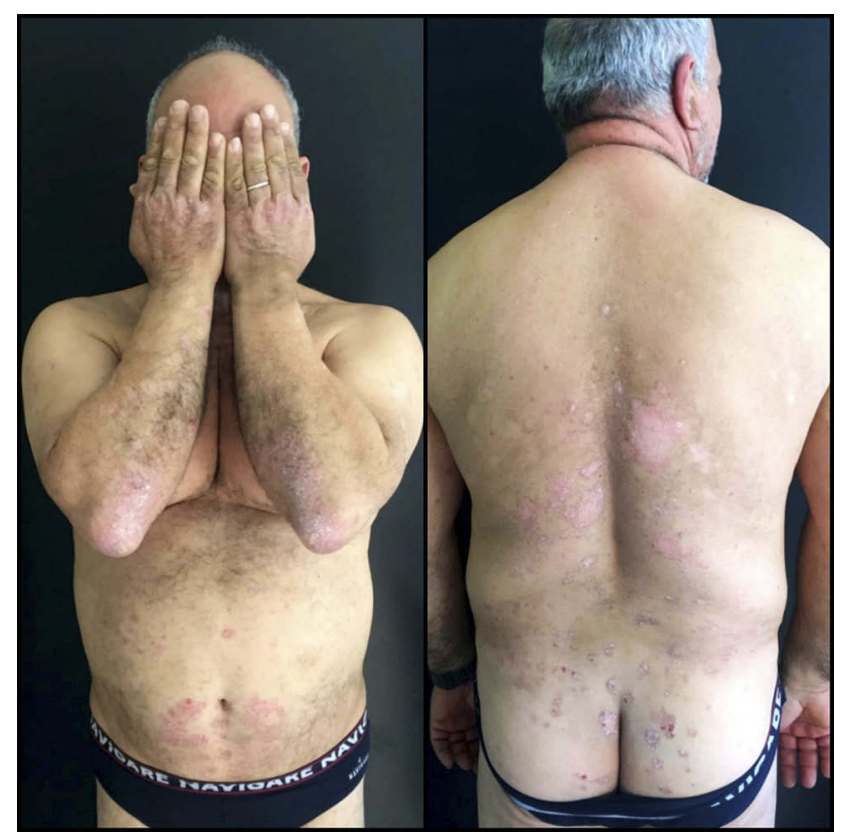

Figure I Patient psoriasis severity before treatment with Apremilast. score of 10 before starting treatment. Diabetes was poorly controlled with a glycemia of $216,00 \mathrm{mg} / \mathrm{dl}$ and a glycate hemoglobin of $8.5 \%$ on the day of the examination despite being medicated with metformin tid and insulin. His BMI was $36.5 \mathrm{Kg} / \mathrm{m} 2$. Regarding the available therapeutic strategies, the patient had poorly controlled dyslipidaemia, thus treatment with Acitretin was contraindicated while cyclosporine was not recommended due to hypertension. Biologic therapies were also contraindicated due to his history of HEV infection. Therefore, we prescribed Apremilast $30 \mathrm{mg}$ twice a day. After 6 months of treatment, he reached an almost complete resolution of his plaque psoriasis (PASI: 1) (Figure 2) with an improvement of the quality of life (DLQI:0) and no evident side effects. Moreover, he lost $30 \mathrm{~kg}$ reaching a BMI of $31 \mathrm{~kg} / \mathrm{m}^{2}$ and had achieved complete control of his diabetes, with a glycemia of $120,00 \mathrm{mg} / \mathrm{dl}$ and a glycate hemoglobin of $6.1 \%$. This allowed him to eliminate insulin from the daily drug routine. Written, informed consent was obtained from the patient with respect to their participation in this case report, including the release of medical information for purposes of research publication. Ethical approval was not required from the institutional review board to publish the details of the case.

\section{Discussion}

In recent years, several studies have shown that patients with psoriasis are at greater risk of diabetes. ${ }^{8}$ The risk of diabetes appears to be higher in patients with long-term

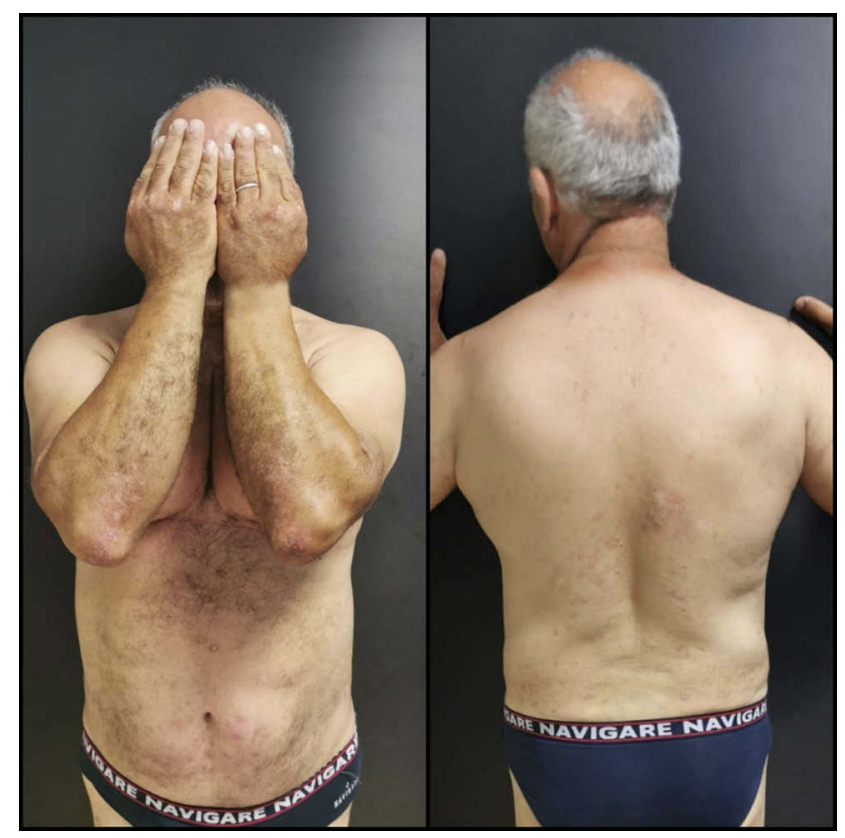

Figure 2 Patient psoriasis severity after 6 months of treatment with Apremilast. 
psoriasis duration and severity, and there seems to be a role between psoriasis severity and the risk of developing diabetes. ${ }^{9}$ Consistent with the increased risk of diabetes, patients with psoriasis seem to have a greater amount of visceral fat. Furthermore, non-alcoholic fatty liver disease (NAFLD) is almost twice as prevalent in patients with psoriasis compared to control patients without psoriasis. This seems noteworthy, as excess liver fat promotes hepatic insulin resistance and NAFLD is a recognized risk factor for diabetes. ${ }^{10}$

Collectively, these results strongly support the fact that obesity and adipose tissue may potentially initiate and guide many of the known inflammatory mechanisms behind the pathogenesis of psoriasis and, finally, could lead to developing diabetes mellitus type 2 .

PDE4 inhibitors, such as Apremilast, elevate intracellular cAMP levels. This leads to a down-regulation of the inflammatory responses by reducing the expression of tumor necrosis factor (TNF)- $\alpha$, interleukin (IL)-23, and other pro-inflammatory cytokines, while increasing antiinflammatory cytokines, such as IL-10. ${ }^{11}$

A recent study has investigated the role of Roflumilast another PDE4 inhibitor - in the treatment of diabetic bladder dysfunction in rats. ${ }^{12}$ The authors reported a reduction in NF$\mathrm{kB}$ gene expression, reduced mRNA expression of TNF-a, IL-6 and IL-1B and lower protein levels of NF-kB, IL-6, IL$1 \mathrm{~B}$ and TNF-a in detrusor smooth muscle strips of mice treated with Roflumilast, showing that PDE4 inhibitors may play a leading role in the reduction of inflammatory mediators in diabetic rats. Moreover, although several studies have already reported fat mass loss in rats as a result of PDE4 inhibitors, ${ }^{13}$ few studies have analyzed this relationship in humans. Only a recent study by Muo et al showed the same percentage of fat mass loss from Roflumilast in humans with prediabetes and overweight/obesity, pointing out novel associations among Roflumilast-induced changes in incretin hormones, inflammatory markers, peripheral insulin sensitivity, and adiposity. $^{14}$

The importance of these studies lies in the additional pharmacological benefits that could be achieved by treating chronic inflammatory diseases such as psoriasis with PDE4-inhibitors due to the improvement of metabolic parameters. This could be of great importance for that cohort of patients suffering from psoriasis and diabetes, as it would allow improvements in both diseases using just one drug, due to its partially common pathogenetic basis.

Moreover, a group of patients able to benefit from a specific drug could be selected. This could allow enable us to fulfill the need of precision medicine and have a more targeted therapy.

\section{Conclusion}

Further studies could clarify the molecular cross-talk between chronic inflammatory diseases and diabetes, with specific regards to the c-AMP/PDE4 pathway, leading to a better understanding of the therapeutic potential of PDE4 inhibitors in patients with psoriasis and cardiovascular comorbidities attributable to a common underlying systemic proinflammatory condition.

\section{Author Contributions}

All authors contributed to data analysis, drafting or revising the article, gave final approval of the version to be published, and agree to be accountable for all aspects of the work.

\section{Disclosure}

The authors report no conflicts of interest in this work.

\section{References}

1. Sawyer LM, Cornic L, Levin LÅ, Gibbons C, Møller AH, Jemec GB. Long-term efficacy of novel therapies in moderate-to-severe plaque psoriasis: a systematic review and network meta-analysis of PASI response. J Eur Acad Dermatol Venereol. 2019;33(2):355-366. doi:10.1111/jdv.15277

2. Augustin M, Krüger K, Radtke MA, Schwippl I, Reich K. Disease severity, quality of life and health care in plaque-type psoriasis: a multicenter cross-sectional study in Germany. Dermatology. 2008;216(4):366-372. doi:10.1159/000119415

3. Ryan C, Kirby B. Psoriasis is a systemic disease with multiple cardiovascular and metabolic comorbidities. Dermatol Clin. 2015;33(1):41-55. doi:10.1016/j.det.2014.09.004

4. Ryan C, Kirby B, Tilg H, et al. Inflammatory pathways and insulin action. Ann Rheum Dis. 2003;27(1):321-328. doi:10.1038/sj. ijo.0802502

5. Wolk K, Sabat R. Adipokines in psoriasis: an important link between skin inflammation and metabolic alterations. Rev Endocr Metab Disord. 2016;17(3):305-317. doi:10.1007/s11154-016-9381-0

6. Gooderham M, Papp K. Selective phosphodiesterase inhibitors for psoriasis: focus on apremilast. BioDrugs. 2015;29(5):327-339. doi:10.1007/s40259-015-0144-3

7. Edwards CJ, Blanco FJ, Crowley J, et al. Apremilast, an oral phosphodiesterase 4 inhibitor, in patients with psoriatic arthritis and current skin involvement: a Phase III, randomised, controlled trial (PALACE 3). Ann Rheum Dis. 2016;75(6):1065-1073. doi:10.1136/ annrheumdis-2015-207963

8. Armstrong AW, Harskamp CT, Armstrong EJ. Psoriasis and the risk of diabetes mellitus: a systematic review and meta-analysis. JAMA Dermatol. 2013;149(1):84-91. doi:10.1001/2013.jamadermatol.406

9. Coto-Segura P, Eiris-Salvado N, González-Lara L, et al. Psoriasis, psoriatic arthritis and type 2 diabetes mellitus: a systematic review and meta-analysis. $\mathrm{Br} \quad J$ Dermatol. 2013;169(4):783-793. doi: $10.1111 /$ bjd. 12473

10. Ganzetti G, Campanati A, Liberati G, Offidani A. Metabolic features in psoriasis. Psoriasis. 2012. doi:10.5772/26129 
11. Houslay MD, Schafer P, Zhang KYJ. Keynote review: phosphodiesterase-4 as a therapeutic target. Drug Discov Today. 2005;10(22):1503-1519. doi:10.1016/S1359-6446(05)03622-6

12. Ding H, Zhang P, Li N, Liu Y, Wang P. The phosphodiesterase type 4 inhibitor roflumilast suppresses inflammation to improve diabetic bladder dysfunction rats. Int Urol Nephrol. 2019;51(2):253-260. doi:10.1007/s11255-018-2038-Z

13. Muo IM, Park S-J, Smith A, et al. Compound D159687, a phosphodiesterase $4 \mathrm{D}$ inhibitor, induces weight and fat mass loss in aged mice without changing lean mass, physical and cognitive function. Biochem Biophys Res Commun. 2018;506(4):1059-1064. doi:10.1016/j.bbrc.2018.10.180
14. Muo IM, MacDonald SD, Madan R, et al. Early effects of roflumilast on insulin sensitivity in adults with prediabetes and overweight/obesity involve age-associated fat mass loss - results of an exploratory study. Diabetes Metab Syndr Obes. 2019;12:743-759. doi:10.2147/ DMSO.S182953

\section{Publish your work in this journal}

Diabetes, Metabolic Syndrome and Obesity: Targets and Therapy is an international, peer-reviewed open-access journal committed to the rapid publication of the latest laboratory and clinical findings in the fields of diabetes, metabolic syndrome and obesity research. Original research, review, case reports, hypothesis formation, expert opinion and commentaries are all considered for publication. The manuscript management system is completely online and includes a very quick and fair peer-review system, which is all easy to use. Visit http://www.dovepress.com/testimonials.php to read real quotes from published authors.

Submit your manuscript here: https://www.dovepress.com/diabetes-metabolic-syndrome-and-obesity-targets-and-therapy-journal 